\title{
Augmented Reality for the Disabled: Review Articles
}

\author{
Ghailan A. Alshafeey ${ }^{1}$, Muhammad Modi Lakulu², M.A. Chyad, Alamoodi \\ Abdullah $^{3}$, Garfan Salem ${ }^{3}$ \\ ${ }^{I}$ Faculty of Arts, Computing and Creative Industry, UPSI; ggylin_alshifay@yahoo.com \\ ${ }^{2}$ Faculty of Arts, Computing and Creative Industry,UPSI; modi@fskik.upsi.edu.my \\ ${ }^{3}$ Faculty of Arts, Computing and Creative Industry, UPSI; Abdullah_alamoodi@outlook.com \\ ${ }^{4}$ Faculty of Arts, Computing and Creative Industry, UPSI; \{Mohammed.chyad1; Salem.garfan\}@gmail.com \\ https://doi.org/10.37134/jictie.vol6.5.2019
}

\begin{abstract}
Augmented Reality or "AR" has been recognized for its significant importance in different areas of technology, not to mention its significant presence in the education sector. This research aimed to map the relevant literature on AR usage with the aim of rehabilitating people with disabilities. The search was performed on three databases: Science Direct, IEEE Explore and Web of Science from 2013 to 2018. . Based on our inclusion and exclusion criteria, $(n=13)$ articles were selected. Seven of the studies $(n=7 / 13)$ intellectual disability. Six of the studies $(n=6 / 6)$ physical disability. The basic idea of this article is to measure the size of the virtual environment utilization in order to rehabilitate the disabled, in addition to the ability of modern technology to restore people with disabilities to normal life and integration into society. This research is expected to display the current literature in this area and to increase the understanding of this research field.
\end{abstract}

Keywords: Augmented Reality (AR), education, disability, student.

\section{INTRODUCTION}

The augmented reality has been defined for its importance and role towards integrating digital information along with user environment in real time settings. Aside from virtual reality settings which create a whole new environment while the AR use the existing one and build on top of it. Augmented reality is a very important tool in different areas of science, particularly, in the rehabilitation of the disabled. The researchers addressed all persons with disabilities, including students of different ages. Disability can be defined as a person's who carry different type of physical or mental deformities due to a partial or total loss of physical, sensory or mental abilities. Statistics have shown that there are approximately 150 million children around the world with special needs. Disability is categorized into two sections: the intellectual disability (ID) and Physical disability. This landscape research consists of two type of disabilities identified. The first section covered the type of disabilities whereby people are suffering from brain deformities or brain diseases that result the disability, for example Stroke Vision, Aphasia, Neglect (VAN), cognitive impairments, cognitive disabilities, autism etc. On the other side, physical disabilities are disabilities related to movement such as weakened hand, hand 
tremors, motor impairments and Loss of limbs. Modern technology, especially the augmented reality, is a promising technology to improve the life style of the rehabilitation of disabled people, especially children, so that they can integrate into society. Vulnerable populations suffer from the unavailability of standardized protocols for patients like children, and also those with intellect disability. It's been shown that many students did not receive sufficient exposure before migrating into the professional working industry (Chuah, Lok, \& Black, 2013). The remaining of the paper discusses different form of disabilities

The major objective for this paper is to shade light on efforts for fellow researchers in response to the use of Augmented Reality in disability rehabilitation, in addition to distinguishing a mapping for research landscape identified in the literature, not to mention the finding out few features that characterize such important research line.

\section{Research Questions}

1 Is there a classification of disabilities in return for the augmented reality?

2 Does the augmented reality suitable to rehabilitate the disabled people?

\section{Research Objectives}

1 To develop map for use augmented reality for disabled people.

2 To investigate whether the augmented reality is useful in helping the disabled to integrate into society.

\section{METHODOLOGY}

This section is meant to describe the article collection process along with the final taxonomy which is the result of dividing the papers into suitable categories.

\section{Data Collection}

Data was collected through three databases, Web of Science (Wos), Science Direct (S.D) and (IEEE). The search was utilized by using query ("Augmented reality" OR AR) AND (Education OR Learning) AND (Disability OR Handicap) AND (Student OR Pupil), the aim was to gather all the data related with disability in the educational settings and to see how relevant it can be with the students. The first screening of the articles resulted in 547 from all the databases. After duplications were removed, 537 articles remained. After that, title and abstract scanning, 403 articles were removed and 134 remained. The last scanning process was full reading which also result in the final set which is 13 articles. 


\section{Inclusion and Exclusion Criteria}

During the screening process, a set of criteria were applied for selecting the relevant studies, 2013 and 2018, an additional criteria was limiting all papers from all databases to English language. Furthermore, in the selection of papers, they were either articles or review articles. There were other aspects of the inclusion related with the topic. The first one was associated with the disability and how AR has been applied in its rehabilitation. There were other types of articles found but were excluded (e.g. conference papers and books) and all papers which don't discuss disability or even papers that discusses three dimensional environments as seen in Error! Reference source not found.

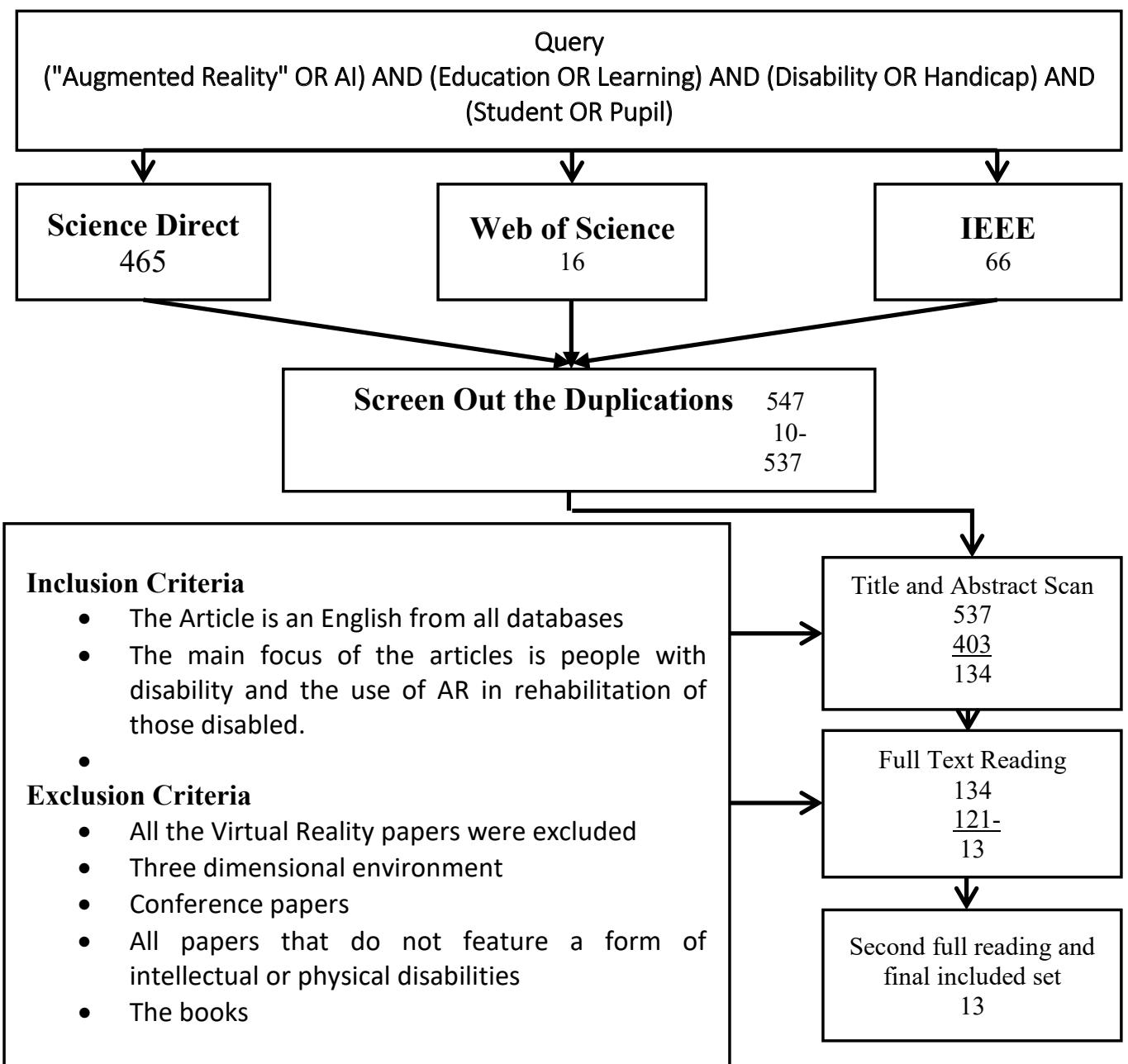

Figure 1: Queries, Protocol, Inclusion and Exclusion Criteria 


\section{Research Landscape}

The landscape displays how different screened articles $(n=13)$ were categorized into two main sections: the first discuss the intellectual disability while the other discuss the physical disability as shown in Error! Reference source not found.

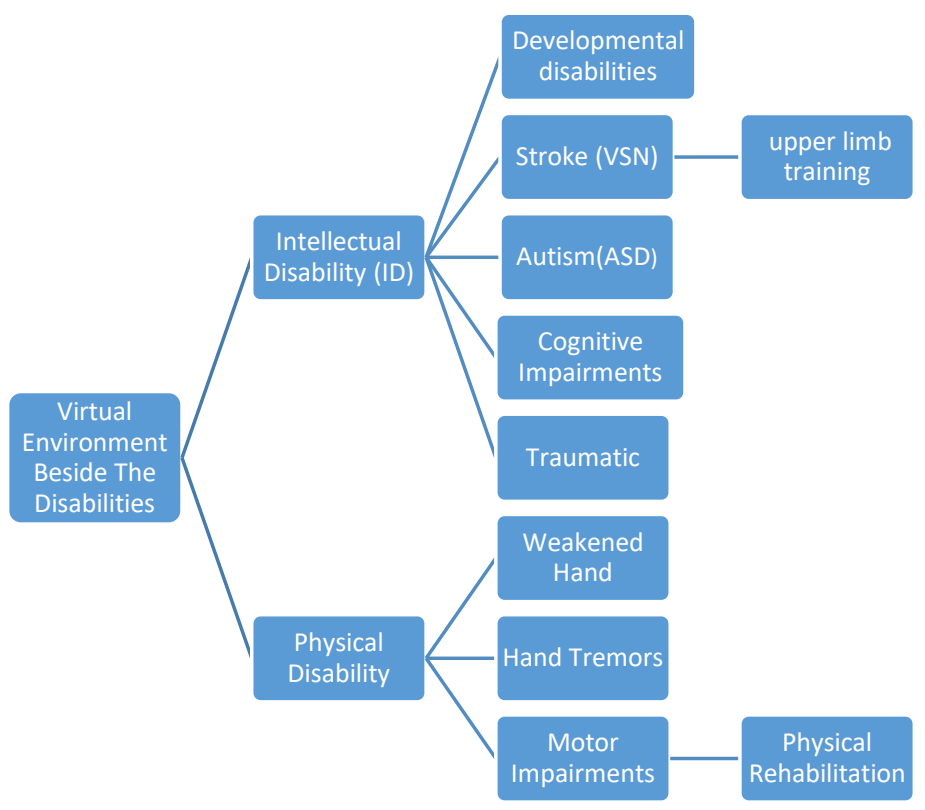

Figure 2: Landscape of Type of Disabilities and Classification

\section{Intellectual Disability (ID)}

Intellectual disability (ID) is a level of mental functioning which is less than average IQ with standard deviation and is accompanied by a marked defect in adaptive behaviour (Deb, Thomas, \& Bright, 2001), which appears in developmental stages from birth to age 18 . The people with mental disabilities need to take greater care more than normal people and the introduction of modern technology may lead to overcoming disability, For some of the populations which suffers from the inexistence for Standardized of patients like the ones with intellectual disability and children. It is been shown that many students did not receive sufficient exposure before migrating into the professional working industry (Chuah, Lok, \& Black, 2013). During the high rate mixed reality exam (MR) learners are able to practice with the exposure to more diversity of children with their families than the ones they previously encountered. Error! Reference source not found. illustrates the use of Virtual child and MR paper. 


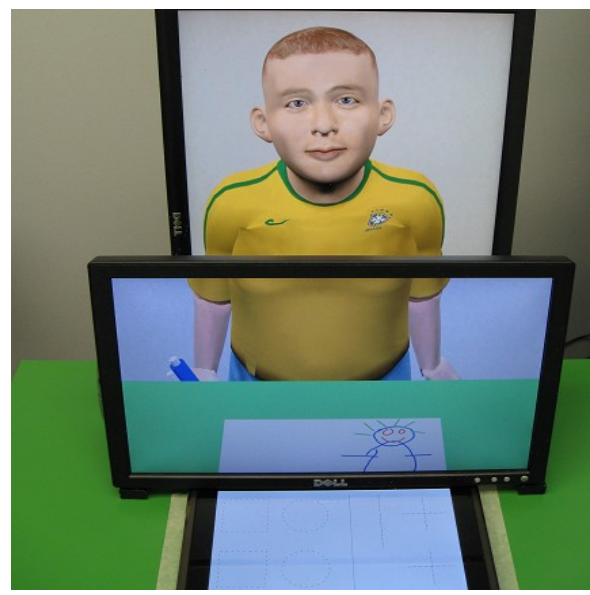

Figure 3: Use of Virtual Child and MR Paper (Chuah, Lok, \& Black, 2013)

\section{a) Developmental Disabilities}

Developmental disability is identified as a diverse group of chronic conditions which are due to impairments that either mental or physical in nature, let alone arising before adulthood (Lin \& Chang, 2015). Children suffers from developmental disabilities often suffer from poor mobility, weak limbs or nerves. They can be helped by using the enhanced reality and focusing on the games that require the child to move his body.it will aid during the integration with the remaining children. The use of these games has proven to be useful, especially the interactive ones. Scratch 2.0 in an Interactive game developed with the aim of to enhancing the strength for children with disabilities bodies motion (Lin \& Chang, 2015). Error! Reference source not found. illustrates the use of interactive games in the virtual environment.

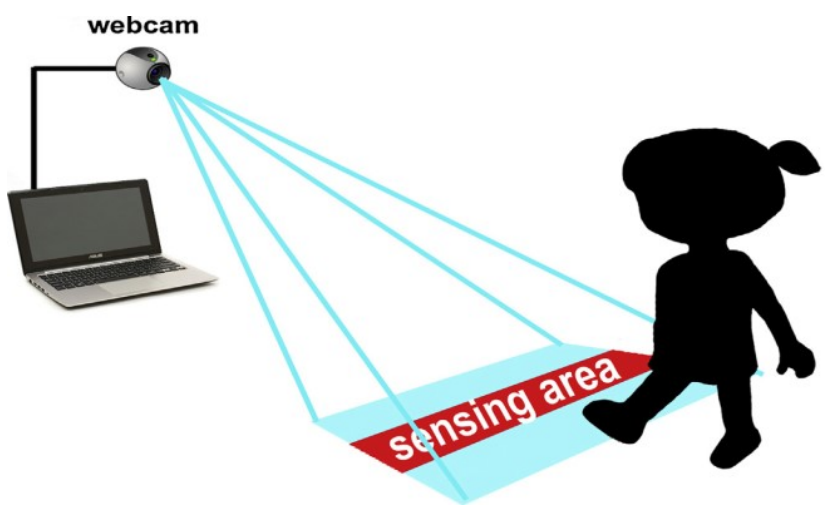


Figure 4: The Concept of the Game Developed in Scratch 2.0 (Lin \& Chang, 2015)

b) Stroke VisuoSpatial Neglect (VSN)

It is a lack of blood flow and nutrition for part of the brain leading to cell death (Cassiday, Lewis, \& Gray, 1998). People suffering from post-stroke neglect (VSN) usually face obstacles during walk. The reason behind the collisions remains unclear whether its occurring due to cognitive deficits which also could be due to VSN or because of an energy deficit after torsion (Aravind, Darekar, Fung \& Lamontagne, 2015). A test group of patients were examined in order to avoid obstacles with the use of joystick and avoiding the motion effect. by used the joystick-drivenvirtual environment-VE in a visor SX60 head mounted display (HMD). A conclusion was that cognitive deficits is due to stroke (VSN). The use of the headset and the virtual environment for stroke patients (VSN) is demonstrated in Error! Reference source not found.

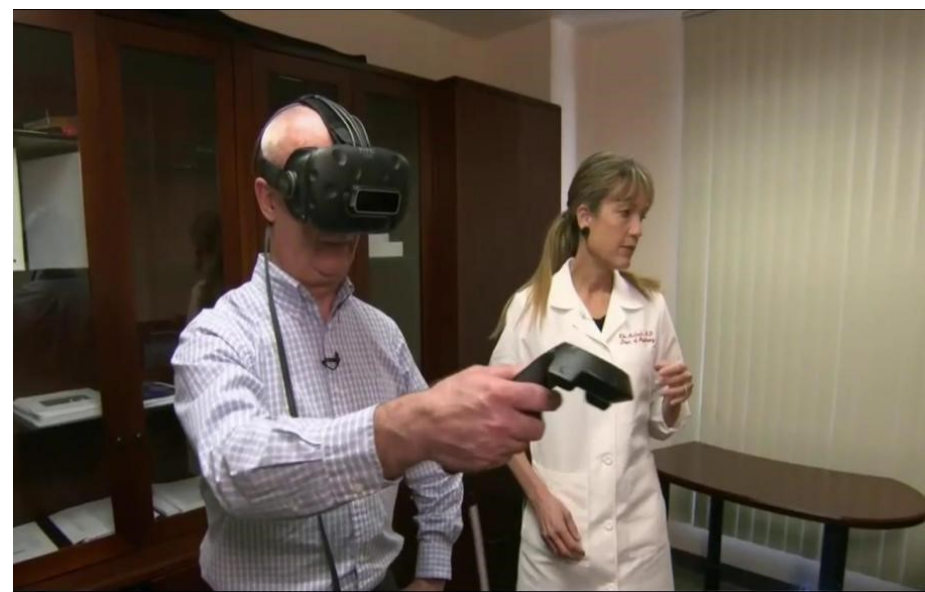

Figure 5: Demonstration for the Use of the Head Set and the Virtual Environment for Stroke Patients (Aravind, Darekar, Fung \& Lamontagne, 2015)

\section{Upper Limb Training (Upper Disorder)}

Is the inability of the person to move the upper limbs due to a specific accident or damage to the brain cells or genetic factor (McAtamney \& Corlett, 1993). Most patients with stroke suffer from paralysis of the upper, lower, or both sides after stroke, which may lead to muscle and nerve atrophy over time if the patient is neglected. It is safe for the utilization of training system based on virtual reality seems to be a motivating training option for after stroke patients. It's important for therapists who use You Grabber in order to be aware of the monitoring for fatigue and be cautious with feedbacks with the use of scores (Lehmann, Baer, \& Scahill, 2007). Error! Reference source not found. demonstrates the method of training upper limbs using virtual reality. 


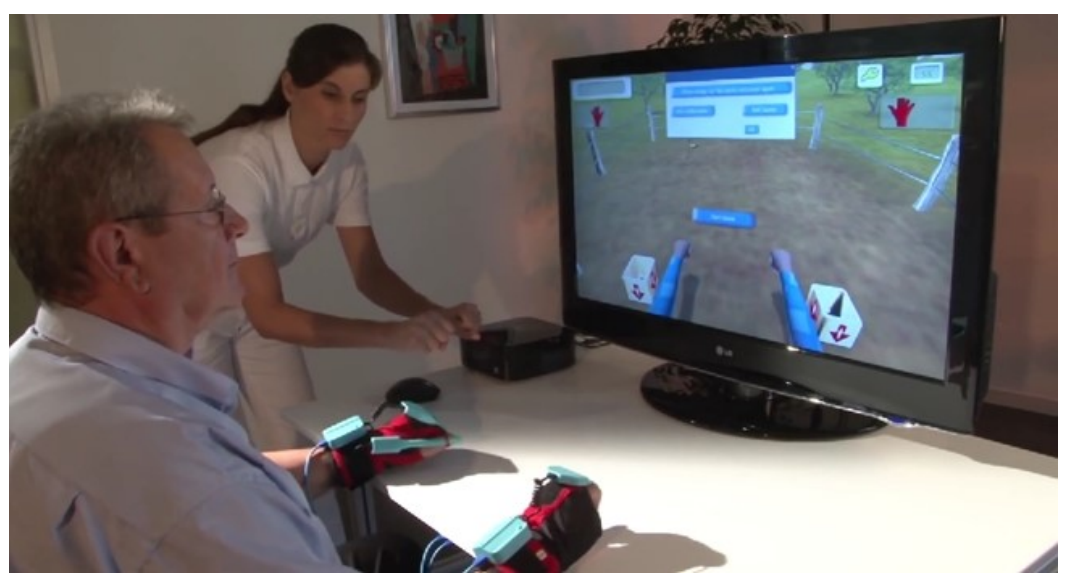

Figure 6: Training Upper Limbs using Augmented Reality (Lehmann, Baer, \& Scahill, 2007)

c) Autism Spectrum Disorder (ASD)

This disorder affects the child's growth and development. It causes weak language and pronunciation of words, weakness in social skills, and interaction with others (White, Keonig, \& Scahill, 2007). Technologies that are based on Augmented Reality (AR) have demonstrated good potential for learning and assessment of children, in addition to adolescents, and adults with autism (Takahashi, Oki, Bourreau, Kitahara, \& Suzuki, 2018).

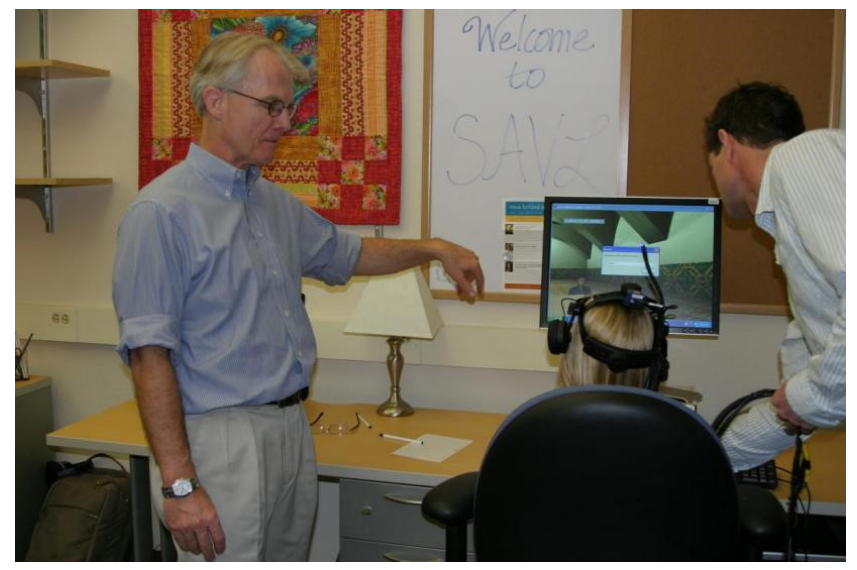

Figure 7: Use Augmented Reality to Treat Children with Autism (Takahashi, Oki, Bourreau, Kitahara, \& Suzuki, 2018) 


\section{d) Cognitive Impairments}

Identified as difficulty while the processing of thoughts which might lead to loss of memory, difficulties in making decision, and the inability to focus and learn issues. A game was created in order with the aim of providing prompts for scenarios associated with recycling, not to mention identifying improper task steps on the fly, and aid users towards learning in order to make amendments (Chang, Kang, \& Liu, 2014; Beidel, et.al., 2017). Error! Reference source not found. is an AR training cognitive impairment.

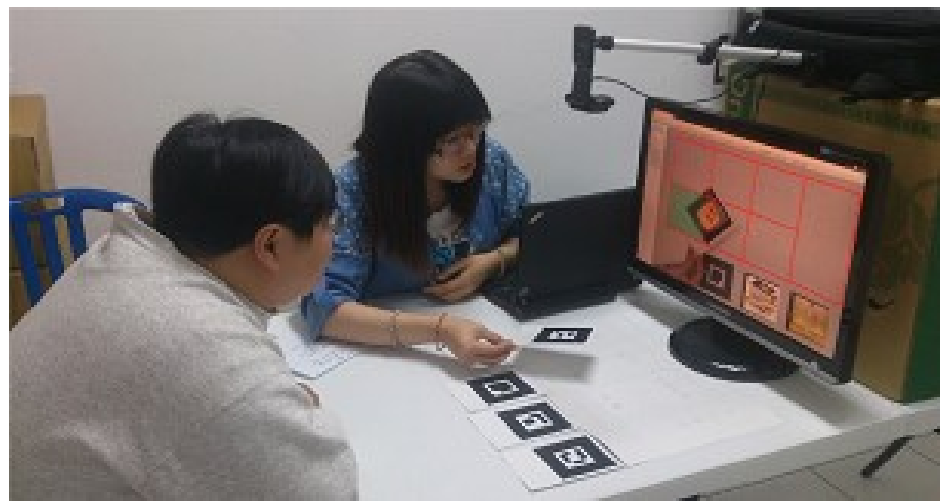

Figure 8: Job Coach (right) and a Participant (left) (Chang, Kang, \& Liu, 2014; Beidel, et.al., 2017)

e) Traumatic (Combat Related PTSD)

It is a particular type of disorder that affects people who have been in combat. augmented reality exposure therapy (ARET) functions by incorporates realistic traumatic cues into exposure therapy to help those with combat-related post-traumatic stress disorder (PTSD) to get treatment (Beidel, et.al., 2017).

\section{Intellectual Disability (ID)}

Is any disability that impairs the physical function of one or more parties or the fine motor skill or motor skill (Janssen, Heymsfield, \& Ross, 2002). It is divided into several sections such as, weakened hand, hand tremors, motor impairments, and physical rehabilitation. An approach was explored for physical rehabilitation named Oculus Rift DK2 which is been marked as one of the best AR technologies (Baldominos, Saez, \& Pozo, 2015). Every screen in the following Figure 9 is projected to an eye by Oculus Rift glasses, producing a 3D effect in the brain. 

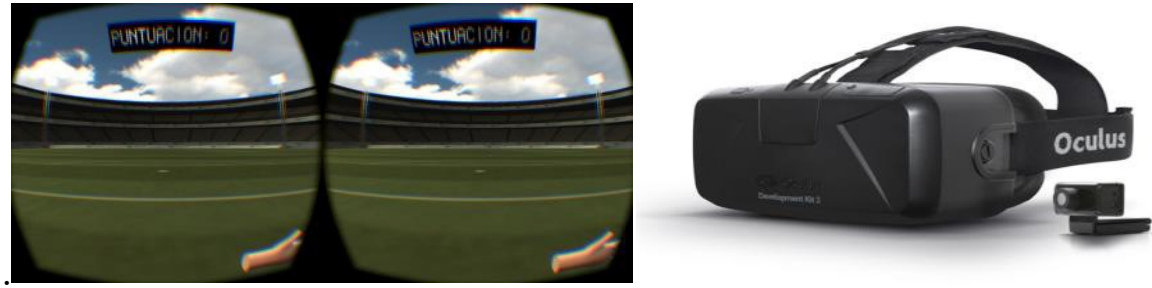

Figure 9: Game Scenario as Seen by the Patient (Baldominos, Saez, \& Pozo, 2015)

\section{a) Weakened Hand}

Weakened hand is the inability of the person to move the hand fully because of atrophy in the nerves or muscle atrophy due to a particular accident or genetic factor or stroke (VSN). Hand rehabilitation learning system, the SAFE Glove, a device that can be used in order to enhance the rehabilitation of subjects with disabilities (Staff, et.al., 2016).The following Error! Reference source not found. is a prototype of the SAFE Glove worn on a hand.

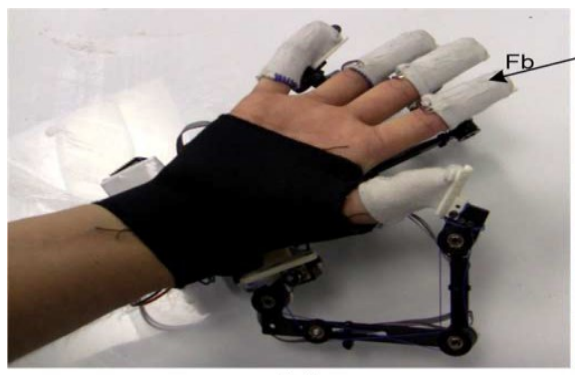

(a)

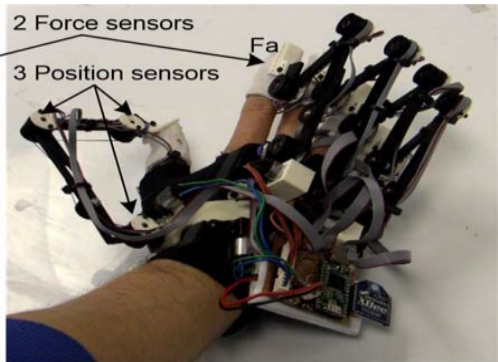

(b)

Figure 10: SAFE Glove Prototype Worn on a Hand (Staff, et.al., 2016)

The hand movement is not controlled due to a satisfactory condition such as a stroke, brain tumor or psychological condition. Using reality system for optical see-through mixed that works by reducing hand tremors so that the individual can type steadily (Wang, Iwai, \& Sato, 2017).

\section{b) Motor Impairments}

It is a general weakness, and fatigue accompanied by various organic symptoms that end to the point of collapse (Ming, Brimacombe, \& Wagner, 2007). It is a chronic fatigue that disrupts the behaviour and membership of the individual. using a 3D computerized game and a peripheral access device which do not need the actions of dragging, clicking, or activating different keys simultaneously were developed (Scardovelli \& Frere, 2015). The following Error! Reference 
source not found. is a snapshot of a 3D game. In the left Window of Figure 11 is the $3 \mathrm{D}$ game, and the control window in the lower right corner (supervisory system).

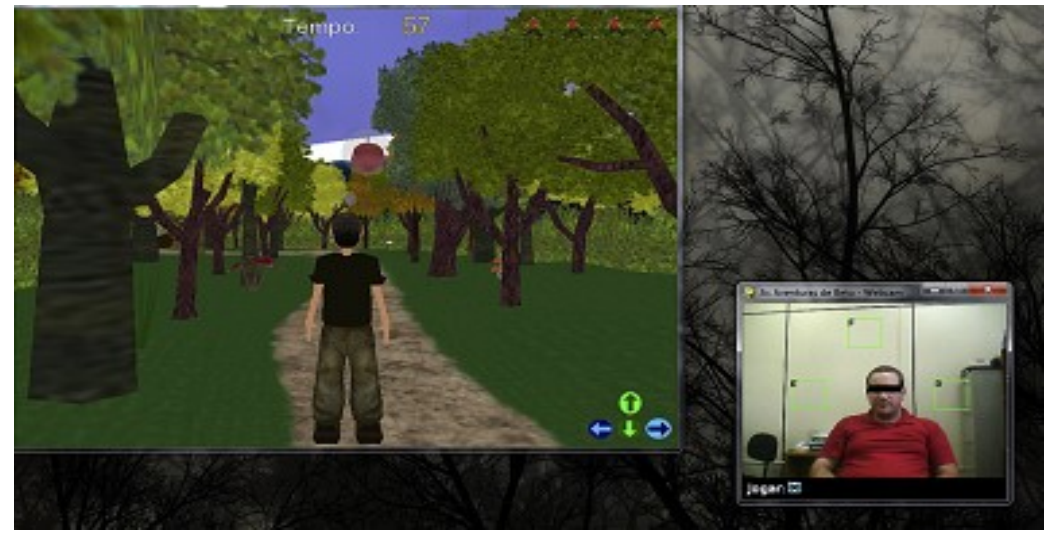

Figure 11: Snapshot of a Supervisory System (Scardovelli \& Frere, 2015)

\section{Deaf}

Is the inability of the affected person to hear temporarily or permanently as a result of genetic factors or environmental or as a result of a particular accident. In order to prompt equal opportunities to all and social inclusion of disabled people is a concern for modern societies around the world, in addition to being a key topic in the European Higher Education agenda, not to mention that it is significance for researchers and academicians (Escudeiro, et.al., 2015).

\section{DISCUSSION}

Through the query search in three databases, we used to search for articles that use augmented reality as a tool to help people with disabilities to live normally, and integrate them into society. The number of related articles found is very small for the number of people with disabilities around the world. We categorized disability types into; (1) mental and (2) physical, and we drew a landscape for all disabilities. After that, we classified disabilities according to the tools used to aid in each disability and how to treat them through the augmented reality. The basic idea of the article is to review the previous studies in the use of the augmented reality in the treatment of the disabled. The results obtained through the query were not satisfactory in term of numbers and experiences which were very low relative to the number of disabled people around the world. It is very important to adapt this modern technology in the rehabilitation of the disabled and people with special needs, especially children with mental and physical disabilities to be able to live normally and exercise their hobby. We recommend more efforts towards using this technology to assist disabled and people with special needs by using larger samples and conducting more augmented reality experiments. 


\section{CONCLUSION}

Various numbers of studies have been identified and surveyed across this landscape. After reviewing the articles on the use of AR in the rehabilitation of disabled persons, we found that the enhanced reality has proved to be useful in various disciplines related to persons with disabilities or those with special needs. We believe that the previous studies are very few in relation to the number of disabled people and the types of disabilities around the world do not rise to the level of ambition. This new technology should be adapted to the rehabilitation of the disabled and people with special needs. It is believed that companies, governments and researchers should consider making more efforts to integrate virtual environments with more types of disabilities that will lead to deep analyses and exploration to help this group of people and experiment with and Use more samples to get deeper results.

\section{ACKNOWLEDGEMENT}

The authors would like to acknowledge Sultan Idris Education University for funding this research. They would also like to acknowledge the help of various anonymous reviewers who provided feedback which helped strengthen the research.

\section{REFERENCES}

Aravind, G., Darekar, A., Fung, J., \& Lamontagne, A. (2015). Virtual reality-based navigation task to reveal obstacle avoidance performance in individuals with visuospatial neglect. IEEE Transactions on Neural Systems and Rehabilitation Engineering. 23, 179-188.

Baldominos, A., Saez, Y., \& del Pozo, C.G. (2015). An approach to physical rehabilitation using state-of-the-art virtual reality and motion tracking technologies. Procedia Computer Science. 64, 10-16.

Beidel, D. C. Frueh, B.C., Neer, S.M., Bowers, C.A., Trachik, B., Uhde, T.W. et al. (2017). Trauma management therapy with virtual-reality augmented exposure therapy for combat-related PTSD: A randomized controlled trial. Journal of Anxiety Disorders.

Cassidy, T.P., Lewis, S., \& Gray, S.C. (21998). Recovery from visuospatial neglect in stroke patients. Journal of Neurology, Neurosurgery \& Psychiatry. 64, 555-557.

Chang, Y.J., Kang, Y. S., \& Liu, F.L. (2014). A computer-based interactive game to train persons with cognitive impairments to perform recycling tasks independently. Research in Developmental Disabilities. 35, 3672-3677.

Chuah, J.H., Lok, B., \& Black, E. (2013). Applying mixed reality to simulate vulnerable populations for practicing clinical communication skills. IEEE Transactions on Visualization and Computer Graphics. 19, 539-546.

Deb, S., Thomas, M., \& Bright, C. (2001). Mental disorder in adults with intellectual disability. 1: Prevalence of functional psychiatric illness among a community-based population aged between 16 and 64 years. Journal of Intellectual Disability Research. 45, 495-505.

Escudeiro, P., Escudeiro, N., Reis, R., Lopes, J., Norberto, M., Baltasar, A.B. et al. (2015). Virtual Sign-A Real Time Bidirectional Translator of Portuguese Sign Language. Procedia Computer Science. 67, 252-262.

Janssen, I., Heymsfield, S.B., \& Ross, R. (2002). Low relative skeletal muscle mass (sarcopenia) in older persons is associated with functional impairment and physical disability. Journal of the American Geriatrics Society. 50, 889-896.

Lehmann, I., Baer, G., \& Schuster-Amft, C. (2107). Experience of an upper limb training program with a non-immersive virtual reality system in patients after stroke: a qualitative study. Physiotherapy.

Lin, C.Y. \& Chang, Y.M. (2015). Interactive augmented reality using Scratch 2.0 to improve physical activities for children 
with developmental disabilities. Research in Developmental Disabilities. 37, 1-8.

McAtamney, L. \& Corlett, E.N. (1993). RULA: A survey method for the investigation of work-related upper limb disorders. Applied Ergonomics. 24, 91-99.

Ming, X., Brimacombe, M., \& Wagner, G.C. (2007). Prevalence of motor impairment in autism spectrum disorders. Brain and Development. 29, 565-570.

Scardovelli, T.A. \& Frère, A.F. (2016). The design and evaluation of a peripheral device for use with a computer game intended for children with motor disabilities. Computer Methods and Programs in Biomedicine. 118, 44-58.

Staff, J., Maggs, J.L., Cundiff, K., \& Evans-Polce, R.J. (2016). Childhood cigarette and alcohol use: negative links with adjustment. Addictive Behaviors. 62,. 122-128.

Takahashi, I., Oki, M., Bourreau, B., Kitahara, I. \& Suzuki, K. (2018). FUTUREGYM: A gymnasium with interactive floor projection for children with special needs. International Journal of Child-Computer Interaction. 15, 37-47.

Wang, K., Iwai, D., \& Sato, K. (2017). Supporting trembling hand typing using optical see-through mixed reality. IEEE Access $5,10700-10708$

White, S.W., Keonig, K., \& Scahill, L. (2007). Social skills development in children with autism spectrum disorders: A review of the intervention research. Journal of Autism and Developmental Disorders. 37, 1858-1868. 